\title{
Small gaps between almost primes, the parity problem and some conjectures of Erdős on consecutive integers
}

\author{
D. A. GOLDSTON, S. W. GRAHAM, J. PINTZ AND C. Y. YILDIRIM
}

\begin{abstract}
In a previous paper, the authors proved that in any system of three linear forms satisfying obvious necessary local conditions, there are at least two forms that infinitely often assume $E_{2}$-values; i.e., values that are products of exactly two primes. We use that result to prove that there are inifinitely many integers $x$ that simultaneously satisfy$$
\omega(x)=\omega(x+1)=4, \Omega(x)=\Omega(x+1)=5 \text {, and } d(x)=d(x+1)=24 .
$$

Here, $\omega(x), \Omega(x), d(x)$ represent the number of prime divisors of $x$, the number of prime power divisors of $x$, and the number of divisors of $x$, respectively. We also prove similar theorems where $x+1$ is replaced by $x+b$ for an arbitrary positive integer $b$. Our results sharpen earlier work of Heath-Brown, Pinner, and Schlage-Puchta.
\end{abstract}

\section{INTRODUCTION}

Erdős had many favorite problems on consecutive integers (see the work of Hildebrand [8]). We will discuss among others the celebrated Erdös-Mirsky conjecture [4] on consecutive values of the divisor function:

Conjecture C1. $d(x)=d(x+1)$ infinitely often.

We will also deal with the analogous conjectures for $\Omega(x)$ and $\omega(x)$, which denote the number of prime factors of a positive integer $x$ counted with and without multiplicity, respectively.

Conjecture C2. $\Omega(x)=\Omega(x+1)$ infinitely often .

Conjecture C3. $\omega(x)=\omega(x+1)$ infinitely often.

The twin prime conjecture is clearly equivalent to a stronger form of Conjectures C1-C2 for $x+2$ in place of $x+1$ namely, with

$$
d(x)=d(x+2)=2, \text { and } \Omega(x)=\Omega(x+2)=1 .
$$

However, the original Conjectures C1-C3 also follow (in a stronger form, similarly to the above) from some analogues of the twin prime conjecture. For example, it is

Date: March 18, 2008.

2000 Mathematics Subject Classification. Primary: 11N37; Secondary 11N36.

The first and second authors were supported in part by NSF Grants, the third author by OTKA grants No. 43623, 49693, 67676 and the Balaton program, the fourth author by TÜBITAK. 
conjectured that $2 p+1$ is prime for infinitely many primes $p$, and Chen's method (see Chapter 11 of [5]) proves that there are infinitely many $p \in \mathcal{P}(\mathcal{P}$ denotes the set of prime numbers) such that either

$$
2 p+1 \in \mathcal{P}
$$

or

$$
2 p+1=p_{1} p_{2} \quad p_{1}, p_{2} \in \mathcal{P}, \quad p_{1} \neq p_{2} .
$$

If, as we believe, (1.3) holds infinitely often, then C1- (3) hold; more precisely

$$
d(2 p)=d(2 p+1)=4, \quad \omega(2 p)=\Omega(2 p)=\omega(2 p+1)=\Omega(2 p+1)=2 .
$$

Due to the connection to the problem in (1.3) ("which is believed to be of the same depth as twin prime conjecture" [8, p. 309]), Conjectures C1-C3 were also considered extremely difficult, if not hopeless.

It was therefore a great surprise for Erdős [3] (and probably for other number theorists as well) when C. Spiro [16] proved in 1981 that

$$
d(x)=d(x+5040) \text { infinitely often. }
$$

At about the same time, Heath-Brown [6] found a conditional proof of $\mathrm{C} 2$ under a hypothesis slightly weaker than the Elliott-Halberstam conjecture [2]. In 1984 he succeeded [7] in proving the original Erdös-Mirsky Conjecture C1 by using the ideas of Spiro in combination of other new ideas. His method also yielded C2, but not C3. About two decades later, J.-C. Schlage-Puchta 14] gave the first proof of C3.

A common feature of all these proofs was their intimate connection with almost primes. More precisely (as suggested by (1.2)-(1.3)), all numbers produced that satisfied the relations C1-C3 had a bounded number of prime factors, but this number (even its parity) was left unspecified by the nature of the methods applied.

The phenomenon which "prevents us from showing that (1.3) has infinitely many solutions" is called the "parity obstacle", "parity barrier" or "parity problem". Selberg gave examples of the two sets [12, Ch. 4]

$$
\mathcal{A}^{(-1)^{r}}(X)=\{a: X<a \leq 2 X, \Omega(a) \equiv r+1 \quad(\bmod 2)\}
$$

which show that the upper and lower bounds obtained under general conditions of the linear sieve of Rosser are optimal. The parity problem is expressed informally as saying that sieve methods cannot differentiate between integers with an even and an odd number of prime factors. Therefore, the parity obstacle prevents the sieve method from revealing the existence of primes in a suitable set as formulated by Greaves [12, p. 171]. For example, while Chen's method yields that $p+2($ or $2 p+1)$ has infinitely often at most two prime factors, the method is unable to specify the parity of the number of prime factors of $p+2$ (or $2 p+1)$. As a result, the seemingly much easier assertion that for infinitely many primes $p, p+2$ (or $2 p+1$ ) has an odd (or even) number of prime factors, is still open [8, p. 310].

The general view about the parity problem and Conjectures C1-C3 3 can be described by again citing the survey paper of Hildebrand [8, p. 310]: "However, there is one 
crucial difference which makes this conjecture [the Erdös-Mirsky conjecture] more accessible than 'twin-prime type' conjectures. Namely, in contrast to the abovementioned problem on the parity of the number of prime factors of $2 p+1$ or $p+2$ when $p$ is prime (and thus, in particular, has an odd number of prime factors), when trying to prove that $d(n)=d(n+1)$ holds infinitely often one does not need to specify the parity of the number of prime factors of $n$. It is this fact that allowed the solution of the Erdős-Mirsky conjecture, while bypassing the deeper problems related to the twin prime conjecture."

The situation is described in exactly the same way by Heath-Brown in his work, where he gives the conditional solution of C2 [6]: "It should however be noted at this point that in solving $\Omega(n)=\Omega(n+1)$ we shall not have specified $\Omega(n)$, or even the parity of $\Omega(n)$. Thus we avoid the parity problem, rather than solve it." In his work [7, where he gives an unconditional solution of C1 and C2, he writes about the proof of (1.5) by Spiro (and his remark refers also for the solution he gives for C1-C2): "Thus one does not know the value of $\Omega(n)$ for the particular $n$ which satisfies $d(n)=d(n+5040)$. In this way one sidesteps the 'parity problem.' "'

In the present work we shall show that the method which yielded the existence of short gaps between primes 11] and $E_{2}$-numbers (numbers with exactly two distinct prime factors) with bounded differences [9, 10] is able to show a stronger variant of Conjectures C1- [3 3 . In this variant, the parity problem is not bypassed but overcome. We can show the above conjectures in the stronger form where the value of the relevant arithmetic function $d, \Omega$ or $\omega$ is specified.

Theorem 1. For any integer $A \geq 4$, there are infinitely many integers $x$ with

$$
\Omega(x)=\Omega(x+1)=A .
$$

Theorem 2. For any integer $A \geq 3$, there are infinitely many integers $x$ with

$$
\omega(x)=\omega(x+1)=A .
$$

Theorem 3. For any positive integer $A$ with $24 \mid A$, there are infinitely many integers $x$ with

$$
d(x)=d(x+1)=A .
$$

As mentioned earlier, Heath-Brown [7] was the first to prove Conjectures C1 and C2, and Schlage-Puchta [14 was the first to prove Conjecture C3. Here, we will give the first proof that there are infinitely many $x$ that simultaneously satisfy the conditions of Conjectures 1 through 3 .

For a positive integer $n$ with prime factorization

$$
n=p_{1}^{\alpha_{1}} p_{2}^{\alpha_{2}} \ldots p_{k}^{\alpha_{k}}
$$

we define the exponent pattern of $n$ to be the multiset $\left\{\alpha_{1}, \alpha_{2}, \ldots, \alpha_{k}\right\}$. We follow the usual conventions for multisets; in particular, an element may appear more than once, and the order of the elements does not matter. This definition is relevant because if $x$ and $x+1$ have the same exponent pattern, then $\omega(x)=\omega(x+1), \Omega(x)=$ $\Omega(x+1)$, and $d(x)=d(x+1)$. We prove 
Theorem 4. There exist infinitely many integers $x$ such that $x$ and $x+1$ both have exponent pattern $\{2,1,1,1\}$. Consequently, there exist infinitely many integers $x$ such that

$$
\omega(x)=\omega(x+1)=4, \quad \Omega(x)=\Omega(x+1)=5, \quad \text { and } d(x)=d(x+1)=24 .
$$

We also note that in fact, we have $f(x)=f(x+1)$ infinitely often for all numbertheoretic functions $f$ with the property that $f(n)$ depends only on the exponent pattern of $n$. Similar comments apply to Theorems 8 and 10 below.

All of the theorems we prove here are done in a relatively straightforward and uniform way. In fact, all of our theorems are simple consequences of the following result proved in [10].

\section{Basic Theorem. We say that}

$$
L_{i}(x)=a_{i} x+b_{i} \quad(1 \leq i \leq 3) \quad a_{i}, b_{i} \in \mathbb{Z}, a_{i}>0,
$$

is an admissible triplet of linear forms if for every prime $p$ there exists $x_{p} \in \mathbb{Z}$ such that

$$
p \nmid L_{1}\left(x_{p}\right) L_{2}\left(x_{p}\right) L_{3}\left(x_{p}\right) .
$$

Let $C$ be any constant. If $\left\{L_{1}, L_{2}, L_{3}\right\}$ is an admissible triplet, then there are two forms $L_{i}(x), L_{j}(x)(i \neq j)$ in the triplet that simultaneously take $E_{2}$-values with both prime factors exceeding $C$ for infinitely many integer values $x$. In other words, there are two forms $L_{i}, L_{j}$ such that

$$
\omega\left(L_{i}(x)\right)=\Omega\left(L_{i}(x)\right)=\omega\left(L_{j}(x)\right)=\Omega\left(L_{j}(x)\right)=2,
$$

and

$$
\left(\prod_{p \leq C} p, L_{i}(x) L_{j}(x)\right)=1
$$

for infinitely many integers $x$.

For future reference, we make one further remark about the admissibility hypothesis in the Basic Theorem. In order for the triplet $\left\{L_{1}, L_{2}, L_{3}\right\}$ to be admissible, it is obviously necessary to have

$$
\left(a_{i}, b_{i}\right)=1, \quad i=1,2,3 .
$$

We shall refer to any system of linear forms that satisfies (1.10) as a reduced system. For any reduced system,

$$
L_{1}(n) L_{2}(n) L_{3}(n) \equiv 0 \quad(\bmod p)
$$

has at most 3 solutions $(\bmod p)$. Therefore, for any reduced system, the admissibility hypothesis is satisfied for any prime $p \geq 5$, and we need check only $p=2$ and $p=3$.

To illustrate how the Basic Theorem can be used, we give a short proof of Theorem 2 in the case $A=3$. Consider the system

$$
L_{1}(m)=6 m+1, L_{2}(m)=8 m+1, L_{3}(m)=9 m+1 .
$$


This system is admissible because we may take $x_{p}=0$ for all primes $p$. We note the relations

$$
4 L_{1}(m)=3 L_{2}(m)+1, \quad 3 L_{1}(m)=2 L_{3}(m)+1, \quad 9 L_{2}(m)=8 L_{3}(m)+1 .
$$

By the Basic Theorem, at least two of the forms will be simultaneously $E_{2}$-numbers for infinitely many values of $x$. We take $C=3$, so that resulting $E_{2}$-numbers have both prime factors exceeding 3 .

Now suppose the two forms giving infinitely many $E_{2}$-numbers are $L_{1}$ and $L_{2}$. Then we let

$$
x=3 L_{2}(m), \quad x+1=4 L_{1}(m) .
$$

If the two relevant forms are $L_{1}$ and $L_{3}$, we let

$$
x=2 L_{3}(m), \quad x+1=3 L_{1}(m) .
$$

If the two relevant forms are $L_{2}$ and $L_{3}$, we let

$$
x=8 L_{3}(m), \quad x+1=9 L_{2}(m) .
$$

In all cases, we obtain infinitely many positive integers $x$ with $\omega(x)=\omega(x+1)=3$.

As the proof illustrates, our approach is to combine the Basic Theorem with a judicious choice of linear forms. Another element of our approach is the idea of "adjoining" extra prime factors. We will discuss this further in Section 2, e.g., see the proof of Theorem 1 .

Next, we consider a modification of Conjectures 1 through 3 with $x, x+1$ replaced by $x, x+2$. As noted before, this is directly related to the twin prime conjecture. We emphasize that while we are unable to specify the parity of $\omega(p+2)$ or $\Omega(p+2)$ for any infinite set of primes $p$, we can specify the common value of $\omega(n)$ and $\omega(n+2)$ (or $\Omega(n)$ and $\Omega(n+2)$ ), where the value can be given almost arbitrarily.

Theorem 5. For any $B \geq 4$ there are infinitely many integers $x$ with

$$
\omega(x)=\omega(x+2)=B .
$$

Theorem 6. For any $B \geq 5$ there are infinitely many integers $x$ with

$$
\Omega(x)=\Omega(x+2)=B .
$$

In the previous papers of this series [9] [10 we investigated the distribution of $E_{2}$ numbers. We are unaware of earlier results (or even earlier known methods capable of yielding such results) on $E_{2}$-numbers showing

$$
\liminf _{n \rightarrow \infty} \frac{q_{n+1}-q_{n}}{\log q_{n} / \log \log q_{n}}=0,
$$

where $q_{n}$ denotes the $n^{\text {th }} E_{2}$ number. This is in contrast to $\mathcal{P}_{2}$-numbers (being the product of at most two primes) where Chen's method proved that we may have infinitely often $n, n+2 \in \mathcal{P}_{2}$. The parity problem seems to indicate that problems with $E_{2}$-numbers may have about the same difficulty as the analogous problems with primes. We proved unconditionally that

$$
\liminf _{n \rightarrow \infty}\left(q_{n+1}-q_{n}\right) \leq 6
$$


in fact, this follows from the Basic Theorem by considering the forms $\{n, n+2, n+6\}$. Although (1.16) is clearly much stronger than (1.15), it is still unclear whether there is any theoretical obstacle which prevents us to find infinitely many pairs of $E_{2}$ numbers with a fixed difference.

Our Theorems 1 6 show, however, that these barriers may be overcome if we increase (slightly) the fixed number of prime divisors. Let $r_{n}$ and $R_{n}$ be the $n^{\text {th }}$ positive integer with

$$
\omega\left(r_{n}\right)=3 \text { and } \Omega\left(R_{n}\right)=3 \text {, respectively. }
$$

An immediate corollary of Theorem 2 is that

$$
\liminf _{n \rightarrow \infty}\left(r_{n+1}-r_{n}\right)=1 .
$$

We also prove

\section{Theorem 7.}

$$
\liminf _{n \rightarrow \infty}\left(R_{n+1}-R_{n}\right) \leq 2
$$

Analogously to (1.17), let $s_{n}$ and $S_{n}$ denote the $n^{\text {th }}$ positive integer with

$$
\omega\left(s_{n}\right)=4 \text { and } \Omega\left(S_{n}\right)=4 \text {, respectively. }
$$

Theorems 1 and 2 imply

$$
\liminf _{n \rightarrow \infty}\left(s_{n+1}-s_{n}\right)=\liminf _{n \rightarrow \infty}\left(S_{n+1}-S_{n}\right)=1 .
$$

The same holds if we consider the sequence $s_{n}^{(\nu)}, S_{n}^{(\nu)}$ of integers defined for an arbitrary integer $\nu \geq 4$ by

$$
\omega\left(s_{n}^{(\nu)}\right)=\nu, \quad \Omega\left(S_{n}^{(\nu)}\right)=\nu .
$$

These results settle almost completely the problem of small differences between consecutive almost primes having exactly $\nu \geq 3$ prime factors (except for the case when prime factors are counted with multiplicity, and $\nu=3$ - see Theorem [7).

We also consider the generalization of Conjectures C1-C3 to an arbitrary shift. In 1997, Pinner [13] used an ingenious extension of Heath-Brown's method to prove that there are infinitely many positive integers $x$ with

$$
d(x)=d(x+n)
$$

for any fixed $n$. His method also works for $\Omega$, but it does not work for $\omega$. However, Buttkewitz [1] has recently proved that $\omega(x)=\omega(x+n)$ holds infinitely often for an infinite set $\mathcal{B}$ of shifts $n$.

We will show that our method provides a positive answer to a stronger form of these more general conjectures as well. Similiarly to Theorems 1 6, we can prescribe the value of the $\omega$ and $\Omega$ functions in case of an arbitrary given shift $n$. Our first results in this direction treat the case of even $n$.

Theorem 8. Let $n$ be an even positive integer. Then there exist infinitely many $x$ such that $x$ and $x+n$ both have exponent pattern

$$
\{2,1,1,1,1\} \text {. }
$$


In particular, there exist infinitely many $x$ such that

$$
\omega(x)=\omega(x+n)=5, \quad \Omega(x)=\Omega(x+n)=6, \quad \text { and } d(x)=d(x+n)=48 .
$$

Theorem 9. If $n \equiv 0(\bmod 4)$, then for any integer $A \geq 4$, there are infinitely many $x$ such that

$$
\Omega(x)=\Omega(x+n)=A .
$$

If $n \equiv 2(\bmod 4)$, then for any integer $A \geq 5$, there are infinitely many $x$ such that

$$
\Omega(x)=\Omega(x+n)=A .
$$

If $n$ is even, then for any positive integer $A \geq 4$, there are infinitely many $x$ such that

$$
\omega(x)=\omega(x+n)=A .
$$

If $n$ is even, then for any positive integer $A$ with $48 \mid A$, there are infinitely many $x$ such that

$$
d(x)=d(x+n)=A
$$

The proof of Theorem 8 uses the linear forms

$$
L_{1}(m)=2 m+n, \quad L_{2}(m)=3 m+n, \quad L_{3}(m)=5 m+n .
$$

In an earlier draft of this paper, we used the same linear forms to show that if $n$ is odd and $n \not \equiv 15(\bmod 30)$, then there are infinitely many $x$ such that $x$ and $x+n$ both have exponent pattern $\{2,1,1,1,1\}$. We eventually realized that one critical property of the forms in (1.22) is that $(3,5)$ is a twin prime pair. We then discovered Theorem 10, which applies to any $n$ satisfying the following

Hypothesis T. Assume that $n$ is an odd positive integer and that there exists a twin prime pair $(p, p+2)$ such that $p(p+2) \nmid n$.

Theorem 10. If $n$ satisfies Hypothesis $T$, then there exist infinitely many positive integers $x$ such that $x$ and $x+n$ both have exponent pattern

$$
\{2,1,1,1,1\} \text {. }
$$

In particular, there are infinitely many $x$ such that

$$
\omega(x)=\omega(x+n)=5, \quad \Omega(x)=\Omega(x+n)=6, \quad \text { and } d(x)=d(x+n)=48 .
$$

By combining Theorem 10 with the trick of adjoining prime factors, we get an analog of Theorem 9 that holds for $n$ satisfying Hypothesis T.

Theorem 11. Assume that $n$ satisfies Hypothesis T. For any positive integer $A \geq$ 5 , there are infinitely many $x$ such that

$$
\omega(x)=\omega(x+n)=A .
$$

For any positive integer $A$ with $48 \mid A$, there are infinitely many $x$ such that

$$
d(x)=d(x+n) .
$$


Along the same lines, we can also prove that $\Omega(x)=\Omega(x+n)=A$ for infinitely many $x$, any $n$ satisfying Hypothesis $\mathrm{T}$, and any $A \geq 6$. However, we will prove a stronger result on $\Omega$ in Theorem 12 .

The previous two theorems lead to the natural problem of determining which integers satisfy Hypothesis $\mathrm{T}$. Take any set $\mathcal{T}_{1}$ with the property that $p \in \mathcal{T}_{1}$ implies that $(p, p+2)$ is a twin prime pair. Let $\mathcal{T}_{2}=\left\{p+2: p \in \mathcal{T}_{1}\right\}, \mathcal{T}=\mathcal{T}_{1} \cup \mathcal{T}_{2}$, and

$$
S=S_{\mathcal{T}}=\prod_{p \in \mathcal{T}} p
$$

Then any $n \not \equiv S(\bmod 2 S)$ satisfies Hypothesis T.

For example, if we take $\mathcal{T}_{1}=\{3,5\}$, we get $S=105$, and so any odd $n$ with $n \not k 105$ (mod 210) satisfies Hypothesis T. Of course, one may take larger sets as well 1 Let (1.26)

$$
\mathcal{T}_{1}=\left\{p: 10^{15}<p \leq 10^{16},(p, p+2) \text { is a twin prime pair }\right\} \text {, and } S=\prod_{p \in \mathcal{T}_{1}} p(p+2) .
$$

According to calculations of Sebah [15] [17, the set $\mathcal{T}_{1}$ has more than $9 \cdot 10^{12}$ elements, and therefore

$$
\log _{10} S>30 \cdot 9 \cdot 10^{12}>2 \cdot 10^{14} .
$$

Consequently, Hypothesis $\mathrm{T}$ is true for any odd $n$ with $n<10^{2 \cdot 10^{14}}$. Moreover, the density of $n$ which fail Hypothesis $\mathrm{T}$ is $\leq 1 /(2 S)$, which is extremely small.

In fact, Hypothesis $\mathrm{T}$ is probably true for all odd $n$, but this appears difficult to prove. The truth of Hypothesis $\mathrm{T}$ for all odd $n$ is equivalent to the existence arbitrarily large sets $\mathcal{T}$, and this in turn is equivalent to the twin prime conjecture. In light of this, it is desirable to give unconditional proofs of (1.23) and (1.24) for all odd $n$.

Theorem 12. Assume that $n$ is an odd positive integer. For any integer $A \geq 5$, there are infinitely many $x$ such that

$$
\Omega(x)=\Omega(x+n)=A .
$$

For any integer $A \geq 6$, there are infinitely many $x$ such that

$$
\omega(x)=\omega(x+n)=A .
$$

For any positive integer $A$ with $288 \mid A$, there are infinitely many $x$ such that

$$
d(x)=d(x+n)=A .
$$

In the proofs of (1.28) and (1.29), we employ the system

$$
L_{1}(m)=672 m+41, \quad L_{2}(m)=672 m+47, \quad L_{3}(m)=672 m+55 .
$$

The Basic Theorem says that at least two of these forms represent $E_{2}$-numbers infinitely often. This provides a usable analogue of the twin prime conjecture. The proof of (1.27) is simpler; it uses the linear forms given in (1.22).

\footnotetext{
${ }^{1}$ Note that $\mathcal{T}_{1}$ and $\mathcal{T}_{2}$ are disjoint if $3 \notin \mathcal{T}_{1}$ and $5 \notin \mathcal{T}_{1}$.
} 


\section{The CAse of CONSECUtive Integers-Theorems 1 Through 7}

For the proof of Theorem 1, we begin with the admissible system

$$
L_{1}(m)=4 m+1, \quad L_{2}(m)=5 m+1, \quad L_{3}(m)=6 m+1,
$$

and we note the relations

$$
5 L_{1}=4 L_{2}+1, \quad 3 L_{1}=2 L_{3}+1, \quad 6 L_{2}=5 L_{3}+1 .
$$

If we apply the Basic Theorem directly to this system, we fail to get the desired result. If, for example, $L_{1}(m)$ and $L_{2}(m)$ are both $E_{2}$ numbers, and if $x=4 L_{2}(m)$, then $\Omega\left(4 L_{2}(m)\right)=4$ and $\Omega\left(5 L_{1}(m)\right)=3$. To remedy this, we modify our system so that $4 m+1$ and $6 m+1$ each have one additional prime factor. We use the Chinese Remainder Theorem to solve the system of congruences

$$
4 m+1 \equiv 0 \quad(\bmod 11), 6 m+1 \equiv 0 \quad(\bmod 7) .
$$

The solution is $m \equiv 8(\bmod 77)$. Accordingly, we take

$$
K(\ell)=77 \ell+8
$$

and we define three new forms $K_{1}, K_{2}, K_{3}$ by setting $r_{1}=11, r_{2}=1, r_{3}=7$, and

$$
K_{i}(\ell)=\frac{L_{i}(K(\ell))}{r_{i}} \quad(i=1,2,3) .
$$

In other words,

$$
K_{1}(\ell)=28 \ell+3, \quad K_{2}(\ell)=385 \ell+41, \quad K_{3}(\ell)=66 \ell+7 .
$$

This is an admissible system; we may use the comment after (1.10) for $p>3$. Otherwise, we let $x_{2}=0$ and $x_{3}=2$. Therefore, we have two forms in (2.4) that are $E_{2}$-numbers with all prime factors exceeding 11 . Consequently, there are at least two relations among

$$
\Omega(4 m+1)=3, \quad \Omega(5 m+1)=2, \quad \Omega(6 m+1)=3,
$$

that are simultaneously true for infinitely many integer values $m$. In view of the relations (2.2) we obtain infinitely many integers $x$ with

$$
\Omega(x)=\Omega(x+1)=4,
$$

since the multiplications in (2.2) adjoin one new prime factor to both $4 m+1$ and $6 m+1$ and two prime factors to $5 m+1$.

For the case $A>4$, we modify the above procedure slightly. We let

$$
r_{1}=11 \cdot 13^{A-4}, \quad r_{2}=17^{A-4}, \quad r_{3}=7 \cdot 19^{A-4} .
$$

and we use the Chinese Remainder Theorem to find $k$ such that

$$
4 k+1 \equiv 0 \quad\left(\bmod r_{1}\right), 5 k+1 \equiv 0 \quad\left(\bmod r_{2}\right), \text { and } 6 k+1 \equiv 0 \quad\left(\bmod r_{3}\right) .
$$

We then take $K(\ell)=r_{1} r_{2} r_{3} \ell+k$, and define the forms $K_{1}, K_{2}, K_{3}$ by

$$
K_{i}(\ell)=\frac{L_{i}(K(\ell))}{r_{i}} \quad(i=1,2,3) .
$$

We apply the Basic Theorem with $C=19$ to the system $\left\{K_{1}, K_{2}, K_{3}\right\}$, and the desired result follows. 
Remark. We will often apply the above procedure of adjoining new prime factors in the following, but we will usually omit the details.

We have already proved Theorem 2 in the case $A=3$; see the discussion beginning with equation (1.11). We do the case $A>3$ by the procedure of adjoining new prime factors. We take $r_{1}, r_{2}, r_{3}$ to be pairwise relatively prime squarefree numbers with exactly $A-3$ prime factors all exceeding 3 , and we start with the system (1.11) as in the case $A=3$.

We will derive Theorem 3 from Theorem 4 , so we now turn our attention to the latter. We begin with the linear forms

$$
L_{1}(m)=3 m+2, \quad L_{2}(m)=4 m+3, \quad L_{3}(m)=10 m+7 .
$$

We note the relations

$$
3 L_{2}=4 L_{1}+1, \quad 3 L_{3}=10 L_{1}+1, \quad 5 L_{2}=2 L_{3}+1,
$$

and we take

$$
r_{1}=5, \quad r_{2}=7^{2}, \quad r_{3}=11^{2}
$$

We see that

$$
L_{i}(m) \equiv 0 \quad\left(\bmod r_{i}\right) \quad(i=1,2,3)
$$

holds for $m \equiv 3956(\bmod 29645)$. Let $K(m)=29645 m+3956$, and consider the three reduced forms

A calculation reveals that

$$
K_{i}(m)=\frac{L_{i}(K(m))}{r_{i}}
$$

$$
K_{1}(m)=17787 m+2374, \quad K_{2}(m)=2420 m+323, \quad K_{3}(m)=2450 m+327 .
$$

The above three forms give an admissible system. To see this, we use the comment after (1.10) for primes $p \geq 5$. For $p=2$ and $p=3$, we note that

$$
5 \cdot 7^{2} \cdot 11^{2} \cdot K_{1} K_{2} K_{3}(1)=L_{1} L_{2} L_{3}(K(1)) \equiv L_{1} L_{2} L_{3}(1) \equiv 1 \quad(\bmod 6),
$$

so we may take $x_{2}=x_{3}=1$. We apply the Basic Theorem and deduce that at least two of these forms are infinitely often simultaneously $E_{2}$-numbers with all prime factors exceeding 11 . If $K_{1}(m)=p_{1} p_{2}$ and $K_{2}(m)=p_{3} p_{4}$, then we may take

$$
x=4 L_{1}(m)=2^{2} \cdot 5 \cdot p_{1} p_{2}, \quad x+1=3 \cdot L_{2}(m)=3 \cdot 7^{2} \cdot p_{3} p_{4} .
$$

The other cases are similar. We conclude that there are infinitely many $x$ such that both $x$ and $x+1$ have exponent pattern

$$
\{2,1,1,1\} \text {. }
$$

This proves Theorem 4 and Theorem 3 in the case $A=24$. When $A=24 B$ with $B>1$, we modify the above with the procedure of adjoining new prime factors. We can do this, for example, by using

$$
r_{1}=5 \cdot 13^{B-1}, \quad r_{2}=7^{2} \cdot 17^{B-1}, \quad r_{3}=11^{2} \cdot 19^{B-1} .
$$

Note that this produces infinitely many $x$ such that both $x$ and $x+1$ have exponent pattern

$$
\{2,1,1,1, B-1\} \text {. }
$$

Consequently, we can say somewhat more; namely, there are infinitely many $x$ with

$$
d(x)=d(x+1)=24 B, \quad \Omega(x)=\Omega(x+1)=B+4, \quad \text { and } \omega(x)=\omega(x+1)=5 .
$$


Theorems 5 and 6 are special cases of Theorem 9 but we give the following independent short proofs. For Theorem 5 in the case $B=4$ and Theorem 6 in the case $B=5$, consider the admissible system

$$
L_{1}(m)=2 m+1, \quad L_{2}(m)=12 m+5, \quad L_{3}(m)=20 m+9 .
$$

Note the relations

$$
12 L_{1}-2 L_{2}=20 L_{1}-2 L_{3}=3 L_{3}-5 L_{2}=2 .
$$

Using the Basic Theorem and adjoining one new prime factor to each of $L_{2}$ and $L_{3}$, we get Theorem 5 for $B=4$. Adjoining two new prime factors to each of $L_{2}$ and $L_{3}$ gives Theorem 6 for $B=5$. The general cases may be done by adjoining further prime factors.

Finally, in order to prove Theorem 7, consider the admissible system

$$
24 m+1, \quad 36 m+1, \quad 72 m+1 .
$$

Using the Basic Theorem and adjoining one new prime factor to the last form we obtain infinitely many positive integers $m$ such that at least two of the relations

$$
\Omega(24 m+1)=2, \quad \Omega(36 m+1)=2, \quad \Omega(72 m+1)=3
$$

hold. Equivalently at least two of the relations

$$
\Omega(72 m+3)=3, \quad \Omega(72 m+2)=3, \quad \Omega(72 m+1)=3
$$

will be true for infinitely many positive integers $m$, and this proves Theorem 7 .

\section{The Case of Even Shift-Theorems 8 and 9}

Throughout this section, we assume that $n$ is even, and we write $n=2 n_{2}$.

We consider the three (non-admissible) forms

$$
L_{1}(m)=2 m+n, \quad L_{2}(m)=3 m+n, \quad L_{3}(m)=5 m+2 n,
$$

with the relations

$$
3 L_{1}=2 L_{2}+n, \quad 5 L_{1}=2 L_{3}+n, \quad 3 L_{3}=5 L_{2}+n .
$$

We will restrict $m$ to some residue class $a(\bmod 60)$, where $a$ depends on $n$,

$$
a \equiv a_{4} \quad(\bmod 4), \quad a \equiv a_{3} \quad(\bmod 3), \quad a \equiv a_{5} \quad(\bmod 5),
$$

and $a_{4}, a_{3}, a_{5}$ are defined as follows. First, we take

$$
a_{4}= \begin{cases}1 & \text { if } n_{2} \text { is even, } \\ n_{2} & \text { if } n_{2} \text { is odd }\end{cases}
$$

In other words, $a_{4} \equiv 3(\bmod 4)$ if $n_{2} \equiv 3(\bmod 4)$ and $a_{4} \equiv 1(\bmod 4)$ otherwise. If $3 \nmid n$, we take $a_{3}=0$. If $3 \mid n$, we write $n=3 n_{3}$ and choose $a_{3}$ so that $a_{3}\left(a_{3}+n_{3}\right) \not \equiv 0$ $(\bmod 3)$. For example, we may take

$$
a_{3}=\left\{\begin{array}{lll}
1 & \text { if } n_{3} \not \equiv 2 \quad(\bmod 3) \\
2 & \text { if } n_{3} \equiv 2 \quad(\bmod 3)
\end{array}\right.
$$


If $5 \nmid n$, we take $a_{5}=0$. If $5 \mid n$, we write $n=5 n_{5}$ and choose $a_{5}$ so that $a_{5}\left(a_{5}+2 n_{5}\right) \not \equiv$ $0(\bmod 5)$. For example, we may take

$$
a_{5}=\left\{\begin{array}{lll}
1 & \text { if } n_{5} \not \equiv 2 \quad(\bmod 5) \\
2 & \text { if } n_{5} \equiv 2 \quad(\bmod 5)
\end{array}\right.
$$

With this choice of $a$ we can show that if $m \equiv a(\bmod 60)$, then

$$
\begin{array}{cl}
2^{1} \| L_{1}(m) \text { if } 4 \mid n, & 2^{2} \| L_{1}(m) \text { if } 4 \nmid n, \\
3^{1} \| L_{2}(m) \text { if } 3 \mid n, & 3 \nmid L_{2}(m) \text { if } 3 \nmid n, \\
5^{1} \| L_{3}(m) \text { if } 5 \mid n, & 5 \nmid L_{3}(m) \text { if } 5 \nmid n .
\end{array}
$$

To prove (3.4), note that if $4 \mid n$, then $2 \mid n_{2}, a_{4}=1$ and

$$
\frac{1}{2} L_{1}(m)=m+n_{2} \equiv a_{4} \equiv 1 \quad(\bmod 2)
$$

If $4 \nmid n$, then $n_{2}$ is odd, and

$$
\frac{1}{2} L_{1}(m)=m+n_{2} \equiv a_{4}+n_{2} \equiv 2 n_{2} \equiv 2 \quad(\bmod 4) .
$$

To prove (3.5), assume first that $3 \mid n$. Then $n=3 n_{3}$, and

$$
\frac{1}{3} L_{2}(m)=m+n_{3} \not \equiv 0 \quad(\bmod 3) \text {. }
$$

If $3 \nmid n$, then $m \equiv 0(\bmod 3)$ and

$$
L_{2}(m)=3 m+n \equiv n \not \equiv 0 \quad(\bmod 3) .
$$

The proof of (3.6) is similar to (3.5), and we leave the details to the reader.

Now let $p_{i}$ be the $i^{\text {th }}$ prime, so that $p_{1}=2, p_{2}=3, p_{3}=5$. Let $b_{1}=b_{2}=1, b_{3}=2$. With this notation,

$$
L_{i}(m)=p_{i} m+b_{i} n
$$

We claim that if $m \equiv a(\bmod 60)$, then

$$
p_{i} \nmid L_{j}(m)
$$

whenever $i \neq j$. For $i=2$ or 3 , this follows because $p_{i}$ divides either $m$ or $n$ but not both. For $p_{1}=2$, this follows because $m$ is odd and $n$ is even.

We rephrase (3.4) through (3.6) by saying that if $m \equiv a(\bmod 60)$, then

$$
2^{\alpha_{1}}\left\|L_{1}(m), \quad 3^{\alpha_{2}}\right\| L_{2}(m), \quad 5^{\alpha_{3}} \| L_{3}(m),
$$

where

$$
\alpha_{1}=\left\{\begin{array}{ll}
1 & \text { if } 4 \mid n, \\
2 & \text { if } 4 \nmid n,
\end{array} \quad \alpha_{2}=\left\{\begin{array}{ll}
1 & \text { if } 3 \mid n, \\
0 & \text { if } 3 \nmid n,
\end{array} \quad \alpha_{3}= \begin{cases}1 & \text { if } 5 \mid n, \\
0 & \text { if } 5 \nmid n .\end{cases}\right.\right.
$$

We adjoin extra factors to the $L_{i}$ according to the following recipe.

If $\left\{\begin{array}{l}4 \mid n \\ 4 \nmid n\end{array}\right.$, then we adjoin a factor of $\left\{\begin{array}{l}19^{2} \\ 19\end{array}\right.$ to $L_{1}$. 
If $\left\{\begin{array}{l}3 \mid n \\ 3 \nmid n\end{array}\right.$, then we adjoin a factor of $\left\{\begin{array}{l}7^{2} \\ 7^{2} \cdot 13\end{array}\right.$ to $L_{2}$.

If $\left\{\begin{array}{l}5 \mid n \\ 5 \nmid n\end{array}\right.$, then we adjoin a factor of $\left\{\begin{array}{l}11^{2} \\ 11^{2} \cdot 17\end{array}\right.$ to $L_{2}$.

By doing this, we have arranged so each $L_{i}$ has a fixed divisor of the form $r_{i} q_{i}^{2}$, where

and

$$
r_{1}=\left\{\begin{array}{ll}
2 & \text { if } 4 \mid n \\
19 & \text { if } 4 \nmid n
\end{array}, r_{2}=\left\{\begin{array}{ll}
3 & \text { if } 3 \mid n \\
13 & \text { if } 3 \nmid n
\end{array}, r_{3}=\left\{\begin{array}{ll}
5 & \text { if } 5 \mid n \\
17 & \text { if } 5 \nmid n
\end{array},\right.\right.\right.
$$

$$
q_{1}=\left\{\begin{array}{ll}
19 & \text { if } 4 \mid n \\
2 & \text { if } 4 \nmid n
\end{array}, q_{2}=7, q_{3}=11 .\right.
$$

We may therefore apply the Basic Theorem to the three forms

$$
K_{i}(\ell)=\frac{L_{i}(60 \ell+a)}{r_{i} q_{i}^{2}}
$$

We obtain at least two different indices $i \in\{1,2,3\}$ such that the numbers in (3.10) would be $E_{2}$-numbers with both prime divisors exceeding 19. Therefore, the numbers $p_{j}\left(p_{i} m+b_{i} n\right)(j \neq i)$ have the exponent-pattern

$$
\{2,1,1,1,1\},
$$

This completes the proof of Theorem 8 ,

Now we consider Theorem 9. Statement (1.21) follows from the construction in Theorem 8 let $A=48 K$ and adjoin a factor of $23^{K-1}$ to the forms $L_{1}, L_{2}, L_{3}$. For the proofs of the other statements, we take $L_{1}, L_{2}, L_{3}$ to be as in (3.1). Then we follow the proof of Theorem 8 down to (3.9). Now let $K(\ell)=60 \ell+a$, and set

$$
K_{i}(\ell)=\frac{L_{i}(K(\ell))}{p_{i}^{\alpha_{i}}}
$$

for $i=1,2,3$. The system $K_{1}, K_{2}, K_{3}$ is admissible, so we may apply the Basic Theorem. By adjoining further primes as necessary, we see that there are infinitely many $x$ and at least two forms with

$$
\omega\left(p_{j} x+b_{j} n\right)=3, \quad \Omega\left(p_{j} x+b_{j} n\right)=2+\alpha_{1},
$$

where $\alpha_{1}$ was defined in (3.9). If we multiply any fixed $p_{j} x+b_{j} n$ by $p_{i}(i \neq j)$, then we obtain two forms with

$$
\omega\left(p_{i}\left(p_{j} x+b_{j} n\right)\right)=4, \quad \Omega\left(p_{i}\left(p_{j} x+b_{j} n\right)\right)=3+\alpha_{1} .
$$

This proves (1.18) with $A=4$, (1.19) with $A=5$, and (1.20) with $A=4$. For larger values of $A$, we adjoin further prime factors as necessary.

\section{Hypothesis T: Proofs of Theorems 10 and 11}

In this section, we assume that $n$ satisfies Hypothesis T. Consequently, $n$ is odd, and there is a twin prime pair $(p, p+2)$ such that $p \nmid n$ or $(p+2) \nmid n$. We divide our argument into two cases depending on whether or not $p=3$. 
Case 1. $p=3$. In this case, we consider the three (non-admissible) linear forms

$$
L_{1}(n)=2 m+n, \quad L_{2}(m)=3 m+n, \quad L_{3}(m)=5 m+2 n .
$$

We note the relations

$$
3 L_{1}-2 L_{2}=5 L_{1}-2 L_{3}=3 L_{3}-5 L_{2}=n .
$$

Our argument for this case is broken into three subcases: (a) $3 \nmid n$ and $5 \nmid n$, (b) $3 \nmid n$ and $5 \mid n$, (c) $3 \mid n$ and $5 \nmid n$.

Subcase 1a. Assume that $3 \nmid n$ and $5 \nmid n$. We choose $m(\bmod 180)$ by the relations

$$
\begin{aligned}
m & \equiv 0 \quad(\bmod 4), \\
m & \equiv 6+5 n \quad(\bmod 9), \\
m & \equiv 0 \quad(\bmod 5) .
\end{aligned}
$$

From the hypotheses, $(n, 30)=1$, so

$$
2 \nmid L_{1}(m), \quad 3 \nmid L_{2}(m), \quad 5 \nmid L_{3}(m) .
$$

Condition (4.3) implies that $m$ is even, so $2 \nmid L_{2}(m)$. However, $n$ is odd, so

$$
L_{3}(m)=5 m+2 n \equiv 2 \quad(\bmod 4),
$$

and $2^{1} \| L_{3}(m)$. Condition (4.4) implies that

$$
L_{1}(m)=2 m+n \equiv 2 n \not \equiv 0 \quad(\bmod 3) \text { and } L_{3}(m)=5 m+2 n \equiv 3 \quad(\bmod 9),
$$

so $3 \nmid L_{1}(m)$ and $3^{1}|| L_{3}(m)$. Finally, condition (4.5) implies that

$$
L_{1}(m) \equiv L_{2}(m) \equiv n \not \equiv 0 \quad(\bmod 5) .
$$

We deduce that $L_{1}, L_{2}$ have no fixed divisors, and $L_{3}$ has the fixed divisors 2 and 3 with exponent one. We adjoin extra factors of $11^{2}$ to $L_{1}$ and $13^{2}$ to $L_{2}$. We apply the Basic Theorem to the reduced forms

$$
\frac{L_{1}(m)}{11^{2}}, \frac{L_{2}(m)}{13^{2}}, \frac{L_{3}(m)}{2 \cdot 3} \text {. }
$$

Multiplying the original forms by 2,3 and 5 according to (4.2), we arrive in all cases at infinitely many pairs $x, x+n$ with exponent pattern $\{2,1,1,1\}$. We adjoin one further prime factor to each form to reach the exponent pattern $\{2,1,1,1,1\}$ enunciated in Theorem 10 .

Subcase 1b. Assume that $3 \nmid n$ and $5 \mid n$. Write $n=5 n_{5}$. In this case, we choose $m(\bmod 180)$ by the relations

$$
\begin{aligned}
m & \equiv 0 \quad(\bmod 4), \\
m & \equiv 6+5 n \quad(\bmod 9), \\
m & \equiv\left\{\begin{array}{llll}
1 & (\bmod 5) & \text { if } n_{5} \not \equiv 2 & (\bmod 5), \\
2 & (\bmod 5) & \text { if } n_{5} \equiv 2 & (\bmod 5) .
\end{array}\right.
\end{aligned}
$$

As in Subcase 1a, we have

$$
2 \nmid L_{1} L_{2}(m), \quad 3 \nmid L_{1} L_{2}(m), \quad 2^{1}|| L_{3}(m), \quad 3^{1}|| L_{3}(m) .
$$

By (4.8), $m\left(m+2 n_{5}\right) \not \equiv 0(\bmod 5)$, thus $5 \nmid L_{1} L_{2}(m)$ and $5^{1}|| L_{3}(m)$. We deduce that $L_{1}, L_{2}$ have no fixed divisors and $L_{3}$ has the fixed divisors $2,3,5$ with exponent 
one. We adjoin factors of $11^{2} \cdot 17$ to $L_{1}$ and $13^{2} \cdot 19$ to $L_{2}$, then we apply the Basic Theorem to the reduced forms

$$
\frac{L_{1}(m)}{11^{2} \cdot 17}, \frac{L_{2}(m)}{13^{2} \cdot 19}, \frac{L_{3}(m)}{2 \cdot 3 \cdot 5} .
$$

Multiplying the original forms by 2, 3 and 5 according to (4.2), we arrive in all cases at numbers with exponent pattern $\{2,1,1,1,1\}$.

Subcase 1c. Assume that $3 \mid n$ and $5 \nmid n$. Write $n=3 n_{3}$. This case is similar to Subcase $1 b$; the primary difference is that the roles of 3 and 5 have been reversed. In this case, we choose $m(\bmod 300)$ by the relations

$$
\begin{aligned}
& m \equiv 2+n \quad(\bmod 4), \\
& m \equiv 10+8 n \quad(\bmod 25) \text {, } \\
& m \equiv\left\{\begin{array}{llll}
1 & (\bmod 3) & \text { if } n_{3} \not \equiv 2 & (\bmod 3) \\
2 & (\bmod 3) & \text { if } n_{3} \equiv 2 & (\bmod 3) .
\end{array}\right.
\end{aligned}
$$

Using condition (4.9), we find that

$$
2 \nmid L_{1} L_{3}(m), \quad 2^{1} \| L_{2}(m) .
$$

Using condition (4.10), we find that

$$
5 \nmid L_{1} L_{3}(m), \quad 5^{1} \| L_{2}(m) .
$$

Using condition (4.11), we find that

$$
3 \nmid L_{1} L_{3}(m), \quad 3^{1}|| L_{2}(m) .
$$

We deduce that $L_{1}$ and $L_{3}$ have no fixed divisors and that $L_{2}$ has the fixed divisors $2,3,5$ with exponent one. We adjoin extra factors of $11^{2} \cdot 17$ to $L_{1}, 13^{2} \cdot 19$ to $L_{3}$, and then we apply the Basic Theorem to the reduced forms

$$
\frac{L_{1}(m)}{11^{2} \cdot 17}, \frac{L_{2}(m)}{2 \cdot 3 \cdot 5} \cdot \frac{L_{3}(m)}{13^{2} \cdot 19},
$$

Multiplying the original forms by 2, 3 and 5 according to (4.2), we arrive in all cases at numbers with exponent pattern $\{2,1,1,1,1\}$.

Case 2. For this case, we assume that Hypothesis $T$ holds for some prime $p>3$. We write $p=2 k+1$, so that

$$
p \equiv k \equiv 2 \quad(\bmod 3) .
$$

In this case, we will use the three linear forms

$$
L_{1}(m)=2 m+n, \quad L_{2}(m)=p m+k n, \quad L_{3}(m)=(p+2) m+(k+1) n .
$$

Note the relations

$$
p L_{1}-2 L_{2}=(p+2) L_{1}-2 L_{3}=p L_{3}-(p+2) L_{2}=n .
$$

We may assume that $3 \cdot 5 \mid n$; otherwise, we may appeal to Case 1 . We will always assume that

$$
m \equiv 1 \quad(\bmod 3) ;
$$

the motivation is that we ensure that $3 \nmid L_{1} L_{2} L_{3}(m)$. 
Subcase 2a. Assume that neither $p$ nor $p+2$ divides $n$. We also assume (4.14), and we specify that

$$
\begin{aligned}
m & \equiv 2+(k+1) n \quad(\bmod 4), \\
L_{3}(m) & \equiv p \quad\left(\bmod p^{2}\right), \\
m & \equiv 0 \quad(\bmod p+2) .
\end{aligned}
$$

From (4.15), we see that

$$
\begin{aligned}
L_{3}(m) & \equiv(p+2) m+(k+1) n \equiv 2 p+(p+3)(k+1) n \\
& \equiv 2+2(k+2)(k+1) n \equiv 2 \quad(\bmod 4) .
\end{aligned}
$$

In the last line, we use the fact that $(k+1)(k+2)$ is always even. Furthermore,

$$
2 m+n \equiv n \equiv 1 \quad(\bmod 2), \quad p m+k n \equiv m+k \equiv(2 k+1) n \equiv 1 \quad(\bmod 2) .
$$

Therefore, $2^{1}|| L_{3}(m)$ and $2 \nmid L_{1} L_{2}(m)$.

From (4.16), we see that $p^{1}|| L_{3}(m)$. Moreover, $p \nmid L_{1} L_{2}(m)$ because

$$
\begin{array}{ll}
L_{1}(m)=2 m+n \equiv-k n \not \equiv 0 & (\bmod p), \\
L_{2}(m)=p m+k n \equiv k n \not \equiv 0 & (\bmod p) .
\end{array}
$$

From (4.17), we see that $L_{i}(m) \equiv b_{i} n(\bmod p+2)$, where $b_{1}=1, b_{2}=k, b_{3}=k+1$. Therefore $(p+2) \nmid L_{1} L_{2} L_{3}(m)$. We conclude that $L_{3}$ has a fixed divisor $2 p$ and $L_{1}, L_{2}$ have no fixed divisors.

Let $p_{1}, p_{2}$ be distinct odd primes with $\left(p_{1} p_{2}, p(p+2) n\right)=1$. We adjoin extra factors of $p_{1}^{2}$ and $p_{2}^{2}$ to $L_{1}$ and $L_{2}$ respectively, then we apply the Basic Theorem to the reduced forms

$$
\frac{L_{1}(m)}{p_{1}^{2}}, \frac{L_{2}(m)}{p_{2}^{2}}, \frac{L_{3}(m)}{2 p} .
$$

Multiplying the original forms by $2, p, p+2$ according to (4.13), we obtain infinitely many positive integers $x$ such that $x$ and $x+n$ both have the exponent pattern $\{2,1,1,1\}$. To get the desired exponent pattern $\{2,1,1,1,1\}$, we adjoin one more prime factor to each form.

Subcase 2b. Assume that $p \nmid n$ and $(p+2) \mid n$, and write $n=(p+2) n_{0}$. We also assume (4.14), and we specify that

$$
\begin{aligned}
m & \equiv 2+3(p+2)(k+1) n \quad(\bmod 4), \\
m & \equiv-(k+1) n_{0}+p \quad\left(\bmod p^{2}\right), \\
m\left(m+(k+1) n_{0}\right) & \equiv 0 \quad(\bmod p+2) .
\end{aligned}
$$

The last condition is possible because there are at most two residue classes mod $(p+2)$ for which (4.20) fails. Furthermore, from (4.18), we see that

$$
\begin{aligned}
L_{3}(m) & =(p+2) m+(k+1) n \equiv 2(p+2)+\left(3(p+2)^{2}+1\right)(k+1) n \\
& \equiv 2 \quad(\bmod 4) .
\end{aligned}
$$

\footnotetext{
${ }^{2}$ We are no longer using the convention that $p_{i}$ denotes the $i^{\text {th }}$ prime.
} 
In the last equation, we use the fact that $j^{2} \equiv 1(\bmod 4)$ whenever $j$ is odd. We also note that

$$
L_{1}(m)=2 m+n \equiv 1 \quad(\bmod 2), \quad \text { and } L_{2}(m)=p m+k n \equiv m+k \equiv 1 \quad(\bmod 2)
$$

Therefore $2^{1}|| L_{3}(m)$ and $2 \nmid L_{1} L_{2}(m)$.

From (4.19), we see that

$$
L_{3}(m) \equiv(p+2)\left(m+(k+1) n_{0}\right) \equiv 2 p \quad\left(\bmod p^{2}\right) .
$$

Furthermore,

$$
L_{1}(m) \equiv-2 k n_{0} \quad(\bmod p) \text { and } L_{2}(m) \equiv k n \quad(\bmod p) .
$$

Therefore $p^{1}|| L_{3}(m)$ and $p \nmid L_{1} L_{2}(m)$. From (4.20), we see that $(p+2) \nmid L_{1} L_{2}(m)$, and

$$
(p+2)^{1}|| L_{3}(m)=(p+2)\left(m+(k+1) n_{0}\right) .
$$

We conclude that $L_{3}$ has fixed divisors $2 p(p+2)$ and $L_{1}, L_{2}$ have no fixed prime divisors.

Let $p_{1}, p_{2}, p_{3}, p_{4}$ be distinct odd primes with $\left(p_{1} p_{2} p_{3} p_{4}, p(p+2) n\right)=1$. We adjoin extra factors of $p_{1}^{2} p_{2}$ and $p_{3}^{2} p_{4}$ to $L_{1}$ and $L_{2}$ respectively, then we apply the Basic Theorem to the reduced forms

$$
\frac{L_{1}(m)}{p_{1}^{2} p_{2}}, \frac{L_{2}(m)}{p_{3}^{2} p_{4}}, \frac{L_{3}(m)}{2 p(p+2)} .
$$

Multiplying the original forms by $2, p, p+2$ according to (4.13), we obtain infinitely many positive integers $x$ such that $x$ and $x+n$ both have the exponent pattern $\{2,1,1,1,1\}$.

Subcase 2c. Assume that $p \mid n$ and $(p+2) \nmid n$, and write $n=p n_{0}$. This case is similar to Subcase $2 \mathrm{~b}$ except that the roles of $p$ and $p+2$ have been reversed. We assume (4.14), and we specify that

$$
\begin{aligned}
m & \equiv 2+3 p k n \quad(\bmod 4), \\
m & \equiv-k n_{0}+p+2 \quad\left(\bmod (p+2)^{2}\right), \\
m\left(m+k n_{0}\right) & \equiv 0 \quad(\bmod p) .
\end{aligned}
$$

From (4.21), we see that

$$
L_{2}(m)=p m+k n \equiv 2 p+\left(3 p^{2}+1\right) k n \equiv 2 \quad(\bmod 4) .
$$

Furthermore,

$$
\begin{aligned}
& L_{1}(m)=2 m+n \equiv 1 \quad(\bmod 2), \text { and } \\
& L_{3}(m)=(p+2) m+(k+1) n \equiv m+k+1 \equiv 1 \quad(\bmod 2) .
\end{aligned}
$$

Therefore $2^{1}|| L_{2}(m)$ and $2 \nmid L_{1} L_{3}(m)$.

From (4.22), we see that

$$
L_{2}(m) \equiv p\left(m+k n_{0}\right) \equiv p(p+2) \quad\left(\bmod (p+2)^{2}\right) .
$$

Furthermore,

$$
\begin{aligned}
& L_{1}(m) \equiv(-2 k-2) n_{0} \quad(\bmod (p+2)), \text { and } \\
& L_{3}(m) \equiv(p-2 k) n_{0} \equiv n_{0} \quad(\bmod (p+2)) .
\end{aligned}
$$


Therefore $(p+2)^{1}|| L_{2}(m)$ and $(p+2) \nmid L_{1} L_{3}(m)$. From (4.23), we see that

$$
p \nmid L_{1} L_{3}(m) \text { and } p^{1} \| L_{2}(m)=p\left(m+k n_{0}\right) .
$$

We conclude that $L_{2}$ has fixed divisors $2 p(p+2)$ and $L_{1}, L_{3}$ have no fixed prime divisors.

Let $p_{1}, p_{2}, p_{3}, p_{4}$ be distinct odd primes with $\left(p_{1} p_{2} p_{3} p_{4}, p(p+2) n\right)=1$. We adjoin extra factors of $p_{1}^{2} p_{2}$ and $p_{3}^{2} p_{4}$ to $L_{1}$ and $L_{3}$ respectively, then we apply the Basic Theorem to the reduced forms

$$
\frac{L_{1}(m)}{p_{1}^{2} p_{2}}, \frac{L_{2}(m)}{2 p(p+2)}, \frac{L_{3}(m)}{p_{3}^{2} p_{4}} .
$$

Multiplying the original forms by $2, p, p+2$ according to (4.13), we obtain infinitely many positive integers $x$ such that $x$ and $x+n$ both have the exponent pattern $\{2,1,1,1,1\}$.

Remark 1. By considering Case 1a and Case 2a, we see that we also have the following result.

Theorem 13. Let $n$ be an odd number. Suppose that there exists a twin prime pair $(p, p+2)$ such that $p \nmid n$ and $(p+2) \nmid n$. Then there are infinitely many positive integers $x$ such that $x$ and $x+n$ both have exponent pattern

$$
\{2,1,1,1\} \text {. }
$$

Note that this generalizes Theorem 4. Note also that using the set $\mathcal{T}_{1}$ from (1.26), we find that the hypothesis of Theorem 13 is true for any odd $n<S^{\prime}$, where

$$
S^{\prime}=\prod_{p \in \mathcal{T}_{1}} p>10^{10^{14}}
$$

\section{The Case of Odd Shift-Proofs of Theorem 12}

We first do the proofs for the results on $\omega$ and $d$; i.e., the proofs of (1.28) and (1.29). The proof of (1.27) is much simpler, and it will be done at the end of this section. For the proofs of (1.28) and (1.29), we will assume that $3 \cdot 5 \cdot 7 \mid n$; otherwise, the desired results follow from Theorem 11 .

Consider the admissible system

$$
672 \ell+41, \quad 672 \ell+47, \quad 672 \ell+55 .
$$

By the Basic Theorem, there are infinitely many positive integers $\ell$ such that at least two of the forms in (5.1) are $E_{2}$-numbers with both prime factors exceeding $C$. In this case, we take $C$ to be the greatest prime factor of $13 n$. In particular,

$$
C \geq 13 \text {. }
$$

We divide our argument into three cases. 
Case 1: $672 \ell+41$ and $672 \ell+47$ are both $E_{2}$-numbers. Let $q=672 \ell+41$, and let $k=112 \ell+7$, so that $q=6 k-1, q \equiv 1(\bmod 4)$, and $k \equiv 3(\bmod 4)$. Consider the system of linear forms

$$
L_{1}(m)=6 m+n, \quad L_{2}(m)=q m+k n, \quad L_{3}(m)=(q+6) m+(k+1) n .
$$

Note that

$$
6 L_{2}-q L_{1}=6 L_{3}-(q+6) L_{1}=(q+6) L_{2}-q L_{3}=n .
$$

Let $q_{1}, q_{2}$ be the prime divisors of $q$. We are assuming $3 \mid n$, and we write $n=3 n_{3}$. We specify that $m$ satisfies the conditions

$$
\begin{array}{rlrl}
m & \equiv 2 \quad(\bmod 4), \\
m\left(2 m+n_{3}\right) & \neq 0 \quad & (\bmod 3), \\
L_{3}(m) & \equiv q_{1} \quad\left(\bmod q_{1}^{2}\right) .
\end{array}
$$

From (5.5), we find that

$$
L_{3}(m)=(q+6) m+(k+1) n \equiv 3 m \equiv 2 \quad(\bmod 4),
$$

and that $2 \nmid L_{1} L_{2}(m)$. From (5.6), we find that

$$
L_{1}(m)=3\left(2 m+n_{3}\right),
$$

and so $3^{1} \| L_{1}(m)$. Moreover, $3 \nmid L_{2} L_{3}(m)$. From (5.7), we find that $q_{1}^{1} \| L_{3}(m)$. Moreover, from (5.4) and (5.7), we see that

$$
-6 L_{1}(m) \equiv 6 L_{2}(m) \equiv n \quad\left(\bmod q_{1}\right) .
$$

Now $q_{1}$ exceeds all prime divisors of $n$, so $q_{1} \nmid L_{1} L_{2}(m)$.

To summarize, our congruence conditions (5.5) through (5.7) imply that $L_{1}$ has fixed divisor $3, L_{2}$ has no fixed divisors, and $L_{3}$ has fixed divisors 2 and $q_{1}$.

Let $p_{1}, p_{2}, p_{3}, p_{4}$ be distinct primes with

$$
\left(p_{1} p_{2} p_{3} p_{4}, 6 q(q+6) n\right)=1 .
$$

We adjoin extra factors of $p_{1}^{2}$ to $L_{1}, p_{2}^{2} p_{3}$ to $L_{2}$, and $p_{4}$ to $L_{3}$. We apply the Basic Theorem to the forms

$$
\frac{L_{1}}{3 p_{1}^{2}}, \frac{L_{2}}{p_{2}^{2} p_{3}}, \frac{L_{3}}{2 p_{4} q_{1}} .
$$

We use the multipliers in (5.4), and we note that

$$
\begin{aligned}
d\left(3 p_{1}^{2} q\right) & =d\left(3 p_{1}^{2}(q+6)\right)=d\left(6 p_{2}^{2} p_{3}\right)=d\left((q+6) p_{2}^{2} p_{3}\right) \\
& =d\left(6 \cdot 2 q_{1} p_{4}\right)=d\left(2 q_{1}^{2} q_{2} p_{4}\right)=24 .
\end{aligned}
$$

We deduce that there are infinitely many $x$ such that

$$
d(x)=d(x+n)=96 .
$$

By the usual procedure of adjoining extra prime factors, we deduce that for any positive integer $A, d(x)=d(x+n)=96 A$ infinitely often. Moreover, we note that

$$
\begin{aligned}
\omega\left(3 p_{1}^{2} q\right) & =\omega\left(3 p_{1}^{2}(q+6)\right)=\omega\left(6 p_{2}^{2} p_{3}\right)=\omega\left((q+6) p_{2}^{2} p_{3}\right) \\
& =\omega\left(6 \cdot 2 q_{1} p_{4}\right)=\omega\left(2 q_{1}^{2} q_{2} p_{4}\right)=4 .
\end{aligned}
$$


We thereby obtain infinitely many $x$ such that $\omega(x)=\omega(x+n)=6$.

Case 2. $672 \ell+41$ and $672 \ell+55$ are both $E_{2}$-numbers. This is similar to Case 1 , so we will leave many of the details. The primary difference is that the prime 7 plays the role played by 3 in Case 1 .

In this case, we let $k=48 \ell+3$, so that $q=14 k-1$ and $k \equiv 3(\bmod 4)$. Consider the system of linear forms

$$
L_{1}(m)=14 m+n, \quad L_{2}(m)=q m+k n, \quad L_{3}(m)=(q+14) m+(k+1) n .
$$

Note that

$$
14 L_{2}-q L_{1}=14 L_{3}-(q+14) L_{1}=(q+14) L_{2}-q L_{3}=n .
$$

Let $q_{1}, q_{2}$ be the divisors of $q$. We are assuming that $7 \mid n$, and we write $n=7 n_{7}$. We specify that $m$ satisfies the conditions

$$
\begin{array}{rlrl}
m & \equiv 2 & (\bmod 4), \\
m\left(2 m+n_{7}\right) & \equiv 0 & (\bmod 7), \\
L_{3}(m) & \equiv q_{1} \quad\left(\bmod q_{1}^{2}\right) .
\end{array}
$$

From these conditions, we find that $2^{1}|| L_{3}(m)$ and $2 \nmid L_{1} L_{2}(m)$. We also find that $7^{1} \| L_{1}(m)$ and $7 \nmid L_{2} L_{3}(m)$. Moreover, $q_{1}^{1} \| L_{3}(m)$ but $q_{1} \nmid L_{1} L_{2}(m)$. In summary, our congruence conditions imply that $L_{1}$ has fixed divisor $7, L_{2}$ has no fixed divisors, and $L_{3}$ has fixed divisors 2 and $q_{1}$, similarly to Case 1 .

Let $p_{1}, p_{2}, p_{3}, p_{4}$ be distinct primes with

$$
\left(p_{1} p_{2} p_{3} p_{4}, 14 q(q+6) n\right)=1 .
$$

We adjoin extra factors of $p_{1}^{2}$ to $L_{1}, p_{2}^{2} p_{3}$ to $L_{2}$ and $p_{4}$ to $L_{3}$. We apply the Basic Theorem to the forms

$$
\frac{L_{1}}{7 p_{1}^{2}}, \frac{L_{2}}{p_{2}^{2} p_{3}}, \frac{L_{3}}{2 p_{4} q_{1}},
$$

and we use the multipliers in (5.10). In this case, we note that (5.8) and (5.9) are true when 6 is replaced by 14 and 3 replaced by 7 . Accordingly, we deduce that for any positive integer $A$, there are infinitely many $x$ such that $d(x)=d(x+n)=96 A$ and $\omega(x)=\omega(x+n)=6$.

Case 3: $672 \ell+47$ and $672 \ell+55$ are both $E_{2}$-numbers. Let $q=672 \ell+47$, and let $k=84 \ell+6$, so that $q=8 k-1 \equiv 3(\bmod 4)$ and $k \equiv 2(\bmod 4)$. Consider the system of linear forms

$$
L_{1}(m)=8 m+n, \quad L_{2}(m)=q m+k n, \quad L_{3}(m)=(q+8) m+(k+1) n .
$$

Note that

$$
8 L_{2}-q L_{1}=8 L_{3}-(q+8) L_{1}=(q+8) L_{2}-q L_{3}=n .
$$

Let $q_{1}, q_{2}$ be the prime divisors of $q$, and choose $m$ so that

$$
\begin{aligned}
m & \equiv 4+(k+1) n \quad(\bmod 8), \\
L_{3}(m) & \equiv q_{1} \quad\left(\bmod q_{1}^{2}\right),
\end{aligned}
$$




$$
L_{3}(m) \equiv q_{2}^{2} \quad\left(\bmod q_{2}^{3}\right) .
$$

From (5.16), we see that

$$
L_{3}(m)=(q+8) m+(k+1) n \equiv-m+(k+1) n \equiv 4 \quad(\bmod 8),
$$

so $2^{2} \| L_{3}(m)$. Note also that $m$ and $n$ are odd and $k$ is even, so $2 \nmid L_{1} L_{2}(m)$. Conditions (5.17) and (5.18) imply that $L_{3}(m)$ has the fixed divisors $2^{2}, q_{1}, q_{2}^{2}$. The forms $L_{1}$ and $L_{2}$ have no fixed divisors, similarly to Cases 1 and 2 .

Let $p_{1}, p_{2}, p_{3}, p_{4}$ be primes with

$$
\left(p_{1} p_{2} p_{3} p_{4}, 2 q(q+8) n\right)=1 .
$$

We add extra divisors $p_{1}^{2} p_{2}^{2}$ to $L_{1}$ and $p_{3}^{2} p_{4}^{2}$ to $L_{2}$. We apply the Basic Theorem to the forms

$$
\frac{L_{1}}{p_{1}^{2} p_{2}^{2}}, \frac{L_{2}}{p_{3}^{2} p_{4}^{2}}, \frac{L_{3}}{2^{2} q_{1} q_{2}^{2}} .
$$

We use the multipliers in (5.15) and note that

$$
\begin{aligned}
d\left(p_{1}^{2} p_{2}^{2} q\right) & =d\left(p_{1}^{2} p_{2}^{2}(q+8)\right)=d\left(8 p_{3}^{2} p_{4}^{2}\right)=d\left(p_{3}^{2} p_{4}^{2}(q+8)\right) \\
& =d\left(2^{3} \cdot 2^{2} q_{1} q_{2}^{2}\right)=d\left(2^{2} q_{1} q_{2}^{2} q\right)=36 .
\end{aligned}
$$

We deduce that there are infinitely many $x$ with

$$
d(x)=d(x+n)=144 .
$$

By adjoining further prime factors, we see that for any positive integer $A, d(x)=$ $d(x+n)=144 A$ infinitely often.

The above argument has to be modified slightly for $\omega$. We take the same linear forms as given in (5.14). As before, we take $m \equiv 4+(k+1) n(\bmod 8)$, and we let $q_{1}, q_{2}$ be the prime divisors of $q$. Let $q_{3}, q_{4}$ be the prime divisors of $q+8$. We choose $m$ so that

$$
L_{2}(m) \equiv q_{3} \quad\left(\bmod q_{3}^{2}\right), \text { and } L_{3}(m) \equiv q_{1} \quad\left(\bmod q_{1}^{2}\right) .
$$

Let $p_{1}, p_{2}$ be as in (5.19). Adjoin an extra factor of $p_{1}$ to $L_{1}$ and an extra factor of $p_{2}$ to $L_{2}$, then apply the Basic Theorem to the forms

$$
\frac{L_{1}}{p_{1}}, \frac{L_{2}}{p_{2} q_{3}}, \frac{L_{3}}{2^{2} q_{1} q_{2}} .
$$

We use the multipliers in (5.15) and note that

$$
\begin{aligned}
\omega\left(p_{1} q\right) & =\omega\left(p_{1}(q+8)\right)=\omega\left(8 p_{2} q_{3}\right)=\omega\left(p_{2} q_{3}(q+8)\right) \\
& =\omega\left(8 \cdot 2^{2} q_{1} q_{2}\right)=\omega\left(2^{2} q_{1} q_{2} q\right)=3 .
\end{aligned}
$$

We deduce that there are infinitely many $x$ such that

$$
\omega(x)=\omega(x+n)=5 .
$$

To make this compatible with Cases 1 and 2, we adjoin one further prime factor so that we obtain infinitely many $x$ with $\omega(x)=\omega(x+n)=6$. This concludes Case 3 .

We can now prove (1.28) and (1.29). From the above three cases, we we see that there are infinitely many $x$ such that $\omega(x)=\omega(x+n)=6$. Statement (1.28) follows from the usual procedure of adjoining further prime factors. For (1.29), we note 
that Cases 1 and 2 give infinitely many $x$ such that $d(x)=d(x+n)=96 A$, while Case 3 gives $d(x)=d(x+n)=144 A$. Statement (1.29) follows upon noting that $\operatorname{lcm}(96,144)=288$.

For the corresponding result on $\Omega$, we return to the simpler procedure used in Section 3. First, we note that by Theorem 13, we have (1.27) with $A=5$ whenever $(n, 15)=1,3$, or 5 . Henceforth, we assume that $15 \mid n$.

We take the forms given in (3.1); i.e.,

$$
L_{1}(m)=2 m+n, \quad L_{2}(m)=3 m+n, \quad L_{3}(m)=5 m+2 n .
$$

We may write $n=3 n_{3}=5 n_{5}$. We specify that

$$
\begin{aligned}
m & \equiv n+2 \quad(\bmod 4), \\
m\left(m+n_{3}\right) & \neq \equiv 0 \quad(\bmod 3), \\
m\left(m+2 n_{5}\right) & \neq \equiv 0 \quad(\bmod 5) .
\end{aligned}
$$

From these conditions, we see that $L_{1}$ has no fixed divisors, $L_{2}$ has fixed divisors 2 and 3 , and $L_{3}$ has fixed divisor 5 . We apply the Basic Theorem to the reduced forms

$$
L_{1}(m), \frac{L_{2}(m)}{2 \cdot 3}, \frac{L_{3}(m)}{5} .
$$

We adjoin one new prime factor to $L_{3}$ and two new prime factors to $L_{1}$. We then find that there are at least two forms $L_{i}$ such that

$$
\Omega\left(L_{i}(r)\right)=4
$$

for infinitely many $r$. Using the relations in (3.2), we find that there are infinitely many $x$ such that

$$
\Omega(x)=\Omega(x+n)=5 .
$$

The completes the proof of (1.27) in the case $A=5$. The general case follows by adjoining further prime factors.

\section{REFERENCES}

[1] Y. Buttkewitz, Master's Thesis, Univ. Freiburg, 2003.

[2] P. D. T. A. Elliott and H. Halberstam, Some applications of Bombieri's theorem, Mathematics 13 (1966), 196-203.

[3] P. Erdős, Some problems on number theory, in: Analytic and elementary number theory (Marseille, 1983), Publ. Math. Orsay, 86-1, 53-57.

[4] P. Erdős and L. Mirsky, The distribution of values of the divisor function $d(n)$, Proc. London Math. Soc. (3) 2 (1952), 257-271.

[5] H. Halberstam and H.-E. Richert, Sieve Methods, Academic Press, London, 1974.

[6] D. R. Heath-Brown, A parity problem from sieve theory, Mathematika 29 (1982), 1-6.

[7] D. R. Heath-Brown, The divisor function at consecutive integers, Mathematika 31 (1984), $141-149$

[8] A. J. Hildebrand, Erdős' problems on consecutive integers, Paul Erdős and his Mathematica I (Bolyai Society Mathematical Studies, 11) Budapest, 2002, pp. 305-317.

[9] D. A. Goldston, S. W. Graham, J. Pintz and C. Y. Yıldırım, Small gaps between primes or almost primes, preprint, arXiv.math.NT/0506067

[10] D. A. Goldston, S. W. Graham, J. Pintz and C. Y. Yıldırım, Small gaps between products of two primes, preprint, arXiv.math.NT/0609615 
[11] D. A. Goldston, J. Pintz and C. Y. Yıldırım, Primes in tuples I, Annals of Mathematics, to appear.

[12] G. Greaves, Sieves in number theory, Springer, Berlin, 2001.

[13] Ch. G. Pinner, Repeated values of the divisor function, Quart. J. Math. Oxford Ser. (2) 48 (1997), no. 192, 499-502.

[14] J.-C. Schlage-Puchta, The equation $\omega(n)=\omega(n+1)$, Mathematika 50 (2003), no. 1-2 (2005), 99-101.

[15] P. Sebah, Counting twin primes and Brun's constant new computation, http://listserv.nodak.edu/archives/nmbrthry.html, August 2002.

[16] C. Spiro, Thesis, Urbana, 1981.

[17] Wolfram Mathworld, http://mathworld.wolfram.com/TwinPrimes.html

D. A. Goldston, Department of Mathematics, San Jose State University, San Jose, CA 95192, USA, e-mail: goldston@math.sjsu.edu

S. W. Graham, Department of Mathematics, Central Michigan University, Mt. Pleasant, MI 48859, USA, email: sidney.w.graham@cmich.edu

J. Pintz, Rényi Mathematical Institute of the Hungarian Academy of Sciences, H-1053 Budapest, Realtanoda u. 13-15. Hungary, e-mail: pintz@renyi.hu

C. Y. Yıldırım, Department of Mathematics, Bog̃aziçi University, Bebek, Istanbul 34342, Turkey, \& Feza Gürsey Enstitüsü Çengelköy, Istanbul, P.K. 6, 81220 Turkey,

e-mail: yalciny@boun.edu.tr 